\title{
Article
}

Subscriber access provided by Universitätsbibliothek Bern

\section{New insights into laser ablation processes of heterogeneous samples: Towards analysis of Through-Silicon-Vias}

Pavel Moreno-García, Valentine Grimaudo, Andreas Riedo, Alena Cedeño López, Reto Wiesendanger, Marek Tulej, Cynthia Gruber, Emanuel Lörtscher, Peter Wurz, and Peter Broekmann

Anal. Chem., Just Accepted Manuscript • DOI: 10.1021/acs.analchem.8b00492 • Publication Date (Web): 03 May 2018

Downloaded from http://pubs.acs.org on May 11, 2018

\section{Just Accepted}

"Just Accepted" manuscripts have been peer-reviewed and accepted for publication. They are posted online prior to technical editing, formatting for publication and author proofing. The American Chemical Society provides "Just Accepted" as a service to the research community to expedite the dissemination of scientific material as soon as possible after acceptance. "Just Accepted" manuscripts appear in full in PDF format accompanied by an HTML abstract. "Just Accepted" manuscripts have been fully peer reviewed, but should not be considered the official version of record. They are citable by the Digital Object Identifier (DOI®). "Just Accepted" is an optional service offered to authors. Therefore, the "Just Accepted" Web site may not include all articles that will be published in the journal. After a manuscript is technically edited and formatted, it will be removed from the "Just Accepted" Web site and published as an ASAP article. Note that technical editing may introduce minor changes to the manuscript text and/or graphics which could affect content, and all legal disclaimers and ethical guidelines that apply to the journal pertain. ACS cannot be held responsible for errors or consequences arising from the use of information contained in these "Just Accepted" manuscripts. 


\title{
New insights into laser ablation processes of heterogeneous samples: Towards analysis of Through-Silicon-Vias
}

\author{
Pavel Moreno-García, ${ }^{\sigma *}$ Valentine Grimaudo, ${ }^{\sigma}$ Andreas Riedo, ${ }^{\rho, \gamma}$ Alena Cedeño López, ${ }^{\sigma}$ Reto \\ Wiesendanger, ${ }^{\rho}$ Marek Tulej, ${ }^{\rho}$ Cynthia Gruber, ${ }^{\Delta}$ Emanuel Lörtscher, ${ }^{\Delta}$ Peter Wurz, ${ }^{\rho}$ Peter Broekmann ${ }^{\sigma *}$ \\ $\sigma$ Department of Chemistry and Biochemistry, University of Bern, Freiestrasse 3, CH-3012 Bern, Switzerland \\ $\rho$ Physics Institute, Space Research and Planetary Sciences, University of Bern, Sidlerstrasse 5, CH-3012 Bern, Switzerland \\ $\gamma$ Sackler Laboratory for Astrophysics, Leiden Observatory, Leiden University, Niels Bohrweg 2, 2333 CA Leiden, The \\ Netherlands \\ $\Delta$ IBM Research - Zurich, Science and Technology Department, Säumerstrasse 4, CH-8803 Rüschlikon, Switzerland.
}

\begin{abstract}
State-of-the-art three-dimensional very large-scale integration (3D-VLSI) relies, among other factors, on the purity of high-aspect-ratio $\mathrm{Cu}$ interconnects such as through-silicon-vias (TSVs). Accurate spatial chemical analysis of electroplated TSV structures has been proven to be challenging due to their large aspect ratios and their multi-material composition $(\mathrm{Cu}$ and $\mathrm{Si})$ with distinct physical properties. Here, we demonstrate that these structures can be accurately analyzed by femto-second (fs) laser beam ablation techniques in combination with ionization mass spectrometry (LIMS). We specifically report on novel preparation approaches for the post-ablation analysis of craters formed upon TSV depth profiling. The novel TSV sample preparation is based on deep and material-selective reactive-ion etching of the Si matrix surrounding the $\mathrm{Cu}$ interconnects thus facilitating systematic focusedion-beam (FIB) investigations of the high-aspect-ratio TSV structures upon ablation. The particular structure of the TSV analyte combined with the $\varnothing_{\text {beam }}>\varnothing_{\mathrm{Cu} \text {-Tsv }}$ condition allowed for an in-depth investigation of fundamental laser ablation processes, particularly focusing on the re-deposition of ablated material at the inner side-walls of the LIMS craters formed. This phenomenon is of imminent importance for the ultimate quantification in any laser ablation-based depth profiling. In addition, we have developed a new method which allows the unambiguous determination of the crossing-point of the $\mathrm{Si} / \mathrm{Cu} \|$ bare $\mathrm{Si}$ interface upon $\mathrm{Cu}$-TSV depth profiling which is based on pronounced, depth-dependent changes in the mass-spectrometric detection of those $\mathrm{Si}_{\mathrm{x}}^{\mathrm{y}+}$ species formed upon the LIMS depth erosion.
\end{abstract}

In recent years, the development of three-dimensional very large-scale integration (3D-VLSI) technologies has significantly emerged to overcome density limitations of classical 2D fabrication schemes, which have already started to deviate from the predicted scaling laws. ${ }^{1}$ Some of the most crucial challenges for 3D-VLSI are related to the signal wiring and transfer, power supply as well as heat removal across densely stacked chips. A viable method to interlink chips in 3DVLSI employs so-called through-silicon-via (TSV) interconnects. TSVs are commonly Cu-based electrical interconnects that are embedded directly into blind holes of the Si wafer. Apart from CVD methods, these metallic interconnects can also be filled by additive-assisted electrodeposition processes. ${ }^{2-4}$ In the latter approach, trace incorporations of the additives into the $\mathrm{Cu}$ matrix might occur, thus negatively influencing the overall TSV performance and its long-term durability. In particular the $\mathrm{Cu}$ grain growth during the post-deposition thermal annealing step might be affected by embedded impurities, ${ }^{5}$ thus resulting in a reduced conductivity of those TSV interconnects. Also it has been observed that the plating-bath chemistry can strongly influence void formation ${ }^{6}$ and the magnitude of $\mathrm{Cu}$ extrusions upon subsequent BEOL (back-end-of-line) processing. ${ }^{7,8}$ To shine light on these phenomena and to ultimately improve the underlying metallization processes, we recently started to investigate the spatial and chemical composition of high aspectratio $\mathrm{Cu}-\mathrm{TSVs}^{9}$ by means of laser ablation ionization mass spectrometry (LIMS) ${ }^{10-15}$ in combination with scanning Auger microscopy (SAM). In that previous work various challenges have been tackled related to i) the multi-component nature of TSVs, e.g., $\mathrm{Cu}$ and $\mathrm{Si}$, with distinct physical properties (thermal conductivity, heat of atomization) relevant to the laser ablation process, and ii) the large vertical $(60 \mu \mathrm{m})$ and confined lateral $(\varnothing=5 \mu \mathrm{m})$ TSV dimensions.

Herein we demonstrate that these structures are also excellent platforms to investigate specific effects in chemical depth profiling that particularly appear when the specimens are analyzed by techniques that employ a laser beam as probing system (LA-ICP-MS, LIBS, LIMS). ${ }^{16-18}$ In practice, we developed a straightforward preparation and analysis method to identify those LIMS depth-profiling measurements in which the laser beam correctly probes the targeted $\mathrm{Cu}$ structures and actually reaches the bottom of the TSVs. By this direct correlation, correct depth profiles can be identified where the TSV was probed throughout the entire LIMS process while non-correct samples with divergent laser positionings can thereby be rejected and disregarded in the subsequent chemical quantification of organic impurities.

In addition, we address in this current paper laser ablation effects which, so far, hampered a true quantification of the TSV LIMS depth profiling and which are particularly related to the re-deposition of ablated material at the inner side-walls of the LIMS craters. For that purpose, chemical analysis of the inner 
crater side-walls was conducted by EDX measurements. These compositional analyses of the crater side-walls became possible due to a novel pre-treatment applied to the TSV samples prior to the LIMS depth profiling. This sample preparation is based on lithographic methods combined with a selective and anisotropic Si etching (Bosch process) thus resulting in Cu-TSV features embedded into well-defined core-shell pillars $(\mathrm{Cu}$ : core; Si: shell). These isolated Si/Cu-TSV pillars served as a starting point for the actual LIMS depth profiling measurements. ${ }^{19}$ Such sample geometry significantly facilitates the subsequent slicing of the isolated specimens by focused ion beam (FIB) milling as basis for the cross-section analyses by SEM and EDX of the LIMS craters formed inside the pillars.

A further experimental challenge we addressed is related to the precise identification of those critical points in the TSV depth profiling processes where the bottom of the $\mathrm{Cu}$ vias is reached. Our previous studies already revealed that it is not sufficient to solely probe the $\mathrm{Cu}$ signal, also due to the above mentioned side-wall re-depositions. We present a novel approach based on mass-spectrometric detection of ionized Si clusters and multiply charged $\mathrm{Si}$ ions whose generation yields were found to drastically change at distinct stages of the TSVs depth profiling process. Hence, they clearly indicate a passing of the $\mathrm{Si} / \mathrm{Cu} \|$ bare $\mathrm{Si}$ interface in the final stage of the TSV depth profiling when reaching the feature bottom and hence can serve as an unambiguous signal tracer.

\section{Experimental section}

\section{Sample preparation}

All samples were provided by BASF SE and consisted of a (quasi)hexagonal array ( $a=210 \mu \mathrm{m}, b=336 \mu \mathrm{m})$ of TSVs $(\varnothing$ $=5 \mu \mathrm{m}, 60 \mu \mathrm{m}$ deep) embedded into a $\mathrm{Si}(100)$ matrix. TSVs and Si matrix are separated from each other by a $650 \mathrm{~nm}$ thick $\mathrm{TOX} / \mathrm{SiO}_{2}$ dielectric layer, a $25 \mathrm{~nm}$ thick TaN/Ta barrier layer, and a $50 \mathrm{~nm}$ thick $\mathrm{Cu}$ seed layer. Prior to the LIMS depth profiling the pristine, Cu-filled TSV wafer coupons $(0.5 \times 0.5$ $\mathrm{cm}^{2}$ ) were mechanically polished from the topside (TSV opening side) to remove the $\mathrm{Cu}$ overburden (excess $\mathrm{Cu}$ material of the electrodeposition process) ${ }^{20,21}$. This removal prevents intermixing of $\mathrm{Cu}$ layers during the TSV LIMS depth profiling. ${ }^{9,22}$ Prior to polishing, the samples were immersed into an epoxy resin upon cold mounting (Struers EpoFix Kit 40200029) and let to cure for $24 \mathrm{~h}$ under ambient conditions. The polishing was carried out in two sequential steps using two wet-polishing paths on which Struers DiaPro Dac 3 and DiaPro Nap B1 diamond suspensions (3 $\mu \mathrm{m}$ diameter, and $1 \mu \mathrm{m}$ diameter, respectively) were poured. 5 min sequential polishing cycles were applied for each suspension using a commercial grinding machine (150 rpm, $\mathrm{P}=10 \mathrm{~N} / \mathrm{m}^{2}$, Struers Tegramin 25) until the samples were overburden-free as inspected by optical microscopy (about $20 \mu \mathrm{m}$ were in fact removed which is more than the overburden). The sample was rinsed after each polishing cycle by deionized water and subsequently dried in an $\mathrm{Ar}$ atmosphere. The resin was finally removed by immersion of the sample in dichloromethane until it was fully detached from the embedding polymer.

To facilitate the preparation of crater cross-sections by means of focused ion beam (FIB) milling, the polished samples were further subjected to standard UV photon lithography techniques using a hexagonally patterned positive tone polymer mask selectively protecting the $\mathrm{Cu}-\mathrm{TSV}$ s and a defined area of the surrounding Si matrix $(\varnothing=50 \mu \mathrm{m}$; AZ4533 resist, spin-coated at $4000 \mathrm{rpm}$ to yield a $3.3 \mu \mathrm{m}$ thick film, backing at $110^{\circ} \mathrm{C}$ on a hot-plate for 60 seconds, $\mathrm{AZ}$ developer to release the exposed areas). A Bosch process of alternating etching/Teflon passivation cycles was applied to achieve $\sim 80 \mu \mathrm{m}$ vertical etching of the unmasked Si surface (Alcatel 1800 Watt, etch rate of $5 \mu \mathrm{m} / \mathrm{min}$ ). The resist was then removed in acetone and the sample was finally cleaned by isopropanol. A schematic representation that summarizes the sample preparation procedure prior to conduction of LIMS analyses and SEM sample images of top view of the sample before and after the polishing step as well as a typical TSV pillar are displayed in Fig. 1.

\section{Laser ablation ionization mass spectrometry (LIMS)}

Laser ablation ionization mass spectrometry depth profiling studies on Si/Cu-TSV pillar samples were carried out using a home-built miniature reflectron-type time-of-flight (TOF) mass analyzer coupled to a Ti-Sapphire laser-system $(\lambda=775 \mathrm{~nm}, \tau$ $\sim 190 \mathrm{fs}$, repetition rate of $1 \mathrm{kHz}$; CPA system, Clark-MXR Inc., USA) to promote the ablation and the ionization of the target material. ${ }^{23-28}$ The figures of merit and the experimental strategy applied herein were similar to those presented in a recent publication, ${ }^{9}$ and only a brief description of the measurements is provided in the following. A more detailed description of the instrument can be found in Refs. 22-27.

Each depth profiling measurement was performed on a fresh $\mathrm{Si} / \mathrm{Cu}-\mathrm{TSV}$ pillar. Each specimen was targeted by the laser system at its center with the help of an $\mathrm{x}-\mathrm{y}-\mathrm{z}$ sample motion stage and the optical microscope implemented inside the vacuum chamber. ${ }^{29}$ Optical micrographs were taken before and after the laser ablation treatment to verify accurate targeting of the features (see e.g., Fig. 2a-d).

The intensity of each detected isotope in the recorded TOF mass spectra was derived from a direct integration method using an adaptive Simpson quadrature integration of the time peaks that are interpolated by a spline function. ${ }^{30}$ Chemical depth profiles were constructed for a set of measurements in which a distinct number of laser shots at different laser pulse energies were applied. The applied laser pulse energies and number of laser shots spanned within the ranges of $E=(1.76-2.47) \mu \mathrm{J}$ and 100 3000 , respectively. Each data point in the chemical depth profiles is the cumulated contribution of detected isotope intensities from 10 single laser pulses that impact the sample surface.

Focused ion beam (FIB) cuts, scanning electron microscopy (SEM) and energy-dispersive X-ray spectroscopy (EDX) Cross sectional FIB-cuts of the Si/Cu-TSV pillars were carried out after the laser ablation experiments by a FEI Helios 450S DualBeam $^{\mathrm{TM}}$ FIB/SEM system with combined electron and ion optics. Ga ions were accelerated to $30 \mathrm{kV}$ with ion currents ranging between 0.23 and $0.79 \mathrm{nA}$ for medium, and of $2.5 \mathrm{nA}$ for high milling rates, respectively. FIB sections were cut in several iterations with intermediate position controlling by acquiring top- and side-view SEM images to confirm whether the $\mathrm{Cu}$ structure had correctly been hit and completely depthprofiled by the laser irradiation (see Fig. 3). Additional FIB-cuts were carried out on non-eroded $\mathrm{Si} / \mathrm{Cu}$-TSV pillars to determine the extent of material removal induced by the polishing procedure. Thus, a high accuracy of the depth scale was ensured. 


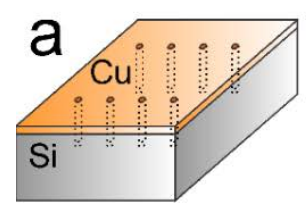

TSV sample as received

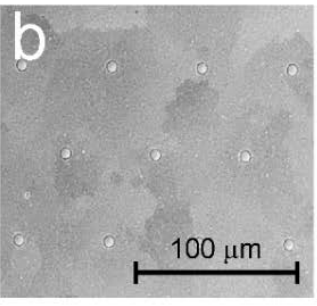

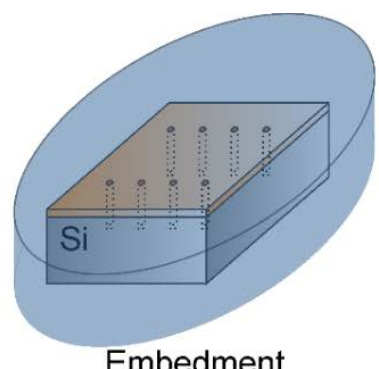

Embedment in resin for polishing

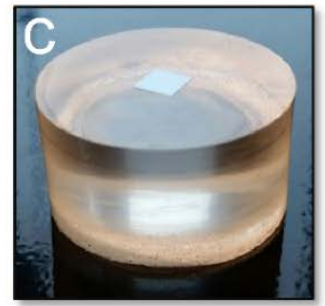

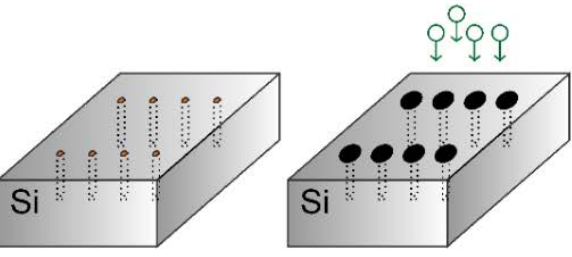

After polishing and resin removal

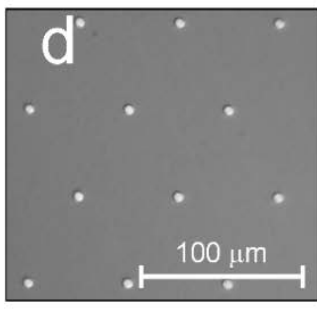

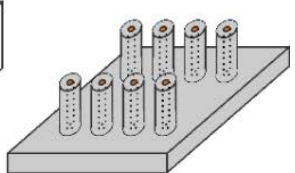

Masking

\& etching

for LIMS

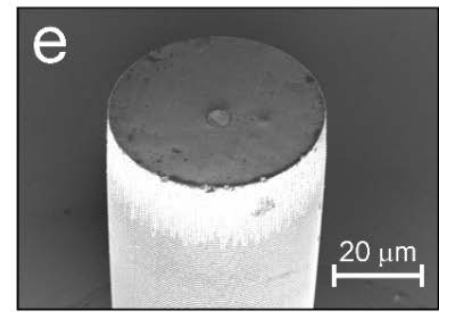

Figure 1: (a) Sketch of Cu-TSV pillars sample preparation steps for LIMS studies. SEM top-view image of sample Cu-TSVs with the overburden before (b), respectively after (d), the polishing preparation steps. (c) Freshly polished Cu-TSV sample embedded in resin. (e) SEM image of a typical TSV pillar after removal of the positive tone polymer mask.

The re-deposition of ablated material on the sidewalls of laserinduced craters was investigated by EDX measurements on selected locations of the crater cross-sections. For that purpose, a Peltier-cooled Roentec EDX detector (type 1106) was used, which was attached to a Raith e_line system (Zeiss column) that served as an SEM with electron acceleration voltages up to 20 $\mathrm{kV}$. A $30 \mu \mathrm{m}$ aperture with beam currents of about $180 \mathrm{pA}$ at $20 \mathrm{kV}$ and spot integration times of 120 seconds was used for EDX analysis by a Bruker Quantax Software.

\section{Results and discussion}

Strategies for the identification of successful and complete targeting of TSVs

Operando microscopy. Most essential to our studies is the accurate targeting of the $\mu \mathrm{m}$-sized Cu-TSV features by the laser probe. Driven by this challenge, an optical microscope was implemented inside the vacuum chamber for online ('operando') monitoring of the laser ablation process and to allow for sample repositioning and adjustments. This feedback control significantly improved the precision of the laser positioning on the sample and along the TSV axis and enabled thereby the further optimization of the ablation parameters. ${ }^{29}$ Figs. 2a and 2c show representative top-down optical micrographs of an isolated core-shell Si/Cu-TSV pillar before, respectively after been irradiated by 3'000 laser shots at a pulse energy of $2.30 \mu \mathrm{J}$. The eroded area (dark imaging contrast in the image center, panel c) is not perfectly symmetric in its outer shape but symmetric with respect to the TSV center. Figs. 2b and 2d show a similar Si/Cu-TSV pillar before, respectively after being irradiated by 5 '000 laser pulses at $1.76 \mu \mathrm{J}$ pulse energy. Here, the TSV was not accurately hit by the center part of the laser beam as it is visible by the slightly shifted center of the eroded area versus the TSV center. The total affected area (dark contrast) extends beyond the TSV lateral domain. In both cases, the applied energy per laser pulse was well above the material specific ablation threshold for $\mathrm{Cu}$ and $\mathrm{Si}$ and the number of pulses was enough to ablate the entire Cu-TSVs. ${ }^{19}$
The orange circles in panels $\mathrm{c}$ and $\mathrm{d}$ serve as guide to the eye indicating the location of the Cu-TSV before being eroded by the laser pulses.

Figs. 2e and $2 \mathrm{f}$ indicate the intensity evolution of the $\mathrm{Cu}^{+}$and $\mathrm{Si}^{+}$signals upon laser ablation which correspond to the crater features presented in Figs. 2c and 2d, respectively. In panel e, the $\mathrm{Cu}^{+}$signal markedly decreases by approximately two orders of magnitude after applying 1'420 laser shots. This characteristic $\mathrm{Cu}^{+}$intensity evolution is clearly indicative for a successful and complete ablation of the Cu-TSV feature. Interestingly, the $\mathrm{Si}^{+}$signal intensity follows, although less pronounced, the same qualitative trend as observed for the $\mathrm{Cu}^{+}$ ions, and slightly decreases when the bottom of the $\mathrm{Cu}-\mathrm{TSV}$ feature is reached. This phenomenon was repeatedly observed for various measurements carried out at different applied pulse energies and number of laser shots. It strongly suggests that the generation of $\mathrm{Si}^{+}$ions is favored or at least assisted by the adjacent $\mathrm{Cu}$ material. ${ }^{19}$

As compared to the graph in Fig. 2e, Fig. 2f shows a completely different intensity evolution with no noticeable decrease of the $\mathrm{Cu}^{+}$signal even after applying about 3 times more laser shots (at $1.76 \mu \mathrm{J}$ ) on the respective Si/Cu pillar (Fig. 2d) than applied on the pillar feature shown in Fig. 2c. This observation seems at first sight counterintuitive and cannot be explained solely by the slightly lower pulse energy used in this experiment. Therefore, it can be assumed that the characteristic steady-state behavior observed in Fig. $2 \mathrm{f}$ and the obvious absence of the $\mathrm{Cu}^{+}$ intensity decrease might be directly correlated to a spatial and lateral offset of the laser beam position with respect to the center location of the TSV feature (Fig. 2d).

From previous studies it is known that ablation 'craters' formed on Si by femtosecond laser irradiation with pulse energies above its ablation threshold exhibit at least two distinct concentric zones/regimes. ${ }^{31}$ The inner one is characterized by the evolving of deep depressions on the targeted material where the actual ablation/removal of material takes place. These ablation processes result from the interaction of the most energetic sections of the laser beam with the target. The outer part, denoted as '(heat) affected' zone ${ }^{32}$ is concentrically 
located surrounding the actual inner crater/depression and is much shallower in the spatial impact. This zone extends over a larger area and is caused by the expanding excitation in the solid beyond the actual volume irradiated by the laser beam. ${ }^{19,31}$
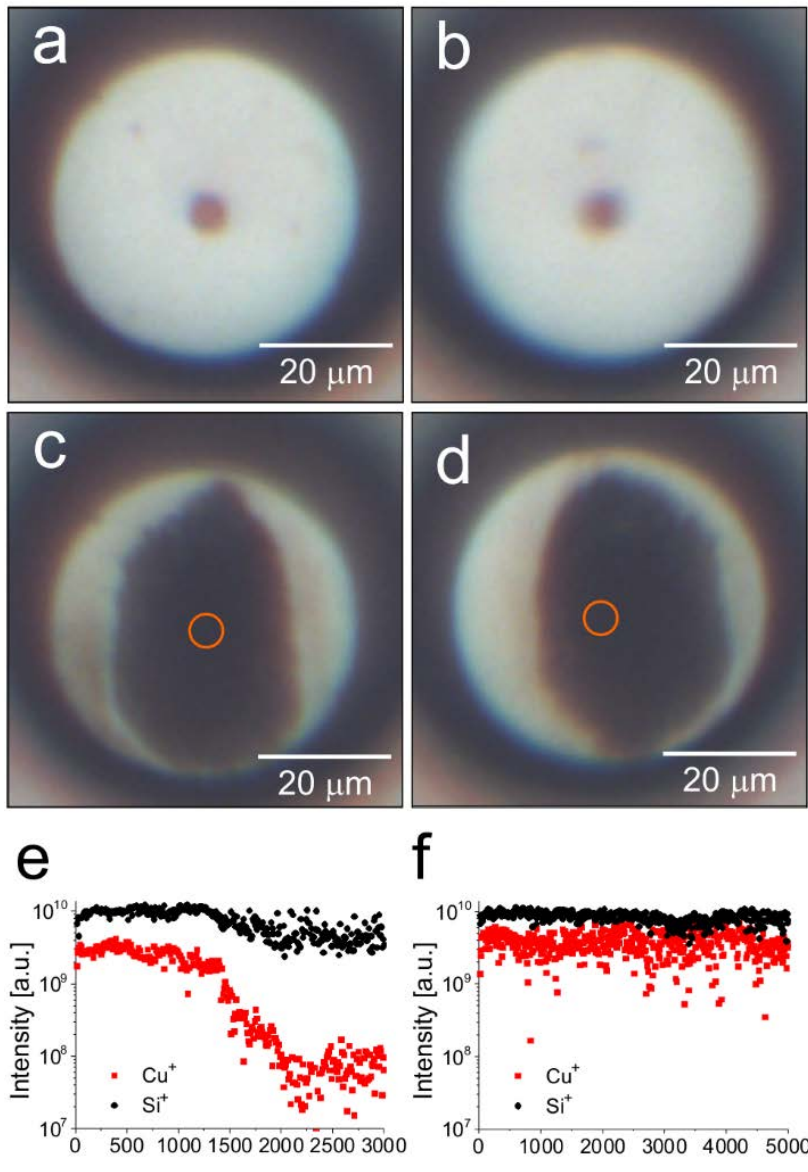

$f$

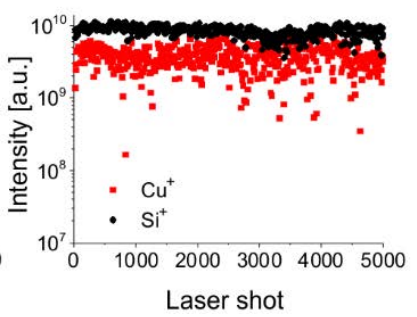

Figure 2: Optical micrographs of Si/Cu-TSV pillars acquired before ( $\mathrm{a}$ and $\mathrm{b}$ ) and after (c: $2.30 \mu \mathrm{J} / \mathrm{pulse}, 3^{\prime} 000$ laser pulses, and d: $1.76 \mu \mathrm{J} /$ pulse, 5'000 laser pulses) laser ablation experiments. (e) and (f) Chemical depth profiles corresponding to the craters shown in panel (c) and (d), respectively. In case of the second depth profile (f) no steep decrease of the Cu signal is observed after 5’000 laser shots as it is expected for a complete removal of the $\mathrm{Cu}$ channel, even.

Re-deposition processes of afore ablated material might additionally contribute to morphological changes taking place not only at the upper side-walls of the actual ablation craters but also in the outer 'heat affected' zone. ${ }^{33}$ Note that the limited lateral resolution of the optical microscope used (Fig. 2) did not allow for the direct visualization of such re-deposited material and the distinction between the actual ablation crater and its corresponding peripheral 'heat affected' zone. Moreover, solely based on the optical inspection presented in Figs. 2a-d, it is not possible to conclude whether or not the TSVs were hit by the center part of the laser beam in a proper manner and throughout the entire ablation process, which, however, is a crucial prerequisite for a successful direct correlation of the TSV depth profiling experiment with MS data. We therefore subjected the depth-profiled Si/Cu-TSV pillars to a FIB milling process to get direct access to the LIMS crater's cross-sections which were then analyzed by high-resolution SEM imaging.
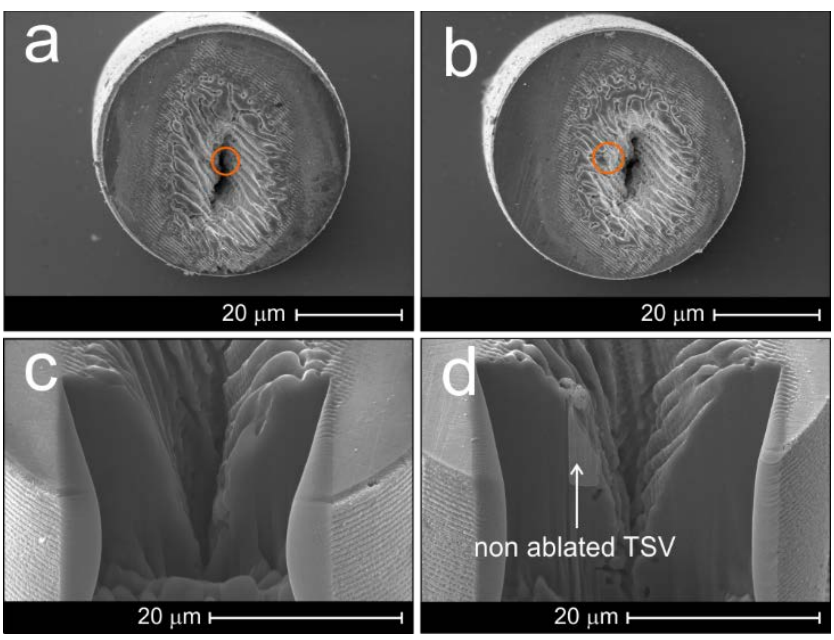

Figure 3: (a) and (b) Top-view SEM images of ablated Si/Cu-TSV pillars shown in Fig. 2c (2.30 $\mu \mathrm{J} /$ pulse, 3'000 laser pulses) and 2d $\left(1.76 \mu \mathrm{J} /\right.$ pulse, $5^{\prime} 000$ laser pulses). The orange circles are guides to the eye indicating where the Cu-TSV was located before the laserinduced ablation. (c) and (d) Corresponding cross-sectional SEM images of sliced pillar samples (a, respectively b). The material erosion by FIB was stopped once the center part of the craters was reached. The cross-sectional SEM images clearly show an incomplete erosion of the Cu-TSV for case (b).

Ex situ FIB/SEM analysis. Figs. 3a and 3b display top-view SEM images of depth-profiled Si/Cu-TSV pillars prior to such FIB treatment. The shown Si/Cu-TSV pillars correspond to the LIMS experiments presented in Fig. 2. Already from this initial top-down SEM inspection (Figs. 3a and 3b) it becomes evident that the lateral dimensions of the actual ablation craters are spatially much more confined inside the crater than it was expected from the initial top-view optical analysis depicted in Fig. 2. Clearly visible in the top-down SEM images are anisotropic ripple-like features that are characteristic for the 'heat affected' zones on Si and surround the actual ablation crater that is visible in the SEM inspection as deep depression. ${ }^{31,34,35}$ Furthermore, our top-down SEM analysis confirms that the Cu-TSV structure (again highlighted by the orange circles) was successfully hit by the central and highly energetic sections of the laser beam only in case of Fig. 3a but not in Fig. 3b. The ablation process therefore resulted in a successful and complete depth profiling of the Cu-TSV only in Fig. 3a but remained incomplete and finally non-successful in Fig. 3b. This is evidenced better by the cross-sectional SEM analyses enabled by the FIB-sliced Si/Cu-TSV pillars shown in Figs. $3 \mathrm{c}$ and $3 \mathrm{~d}$. While the Si section surrounding the ablation crater appears to be homogenous in Fig. 3c, Fig. 3d shows a residual of the Cu-TSV which is clearly visible by the material contrast in vertical direction. These experimental findings explain the abnormal depth profiles in Fig. $2 \mathrm{f}$ and in particular the absence of an abrupt decrease of the $\mathrm{Cu}^{+}$signal intensity as a section of the laser beam still ablated $\mathrm{Cu}$ during the entire process. Such a drop down in the $\mathrm{Cu}^{+}$signal intensity is therefore considered indicative for reaching the TSV's bottom in the course of the depth profiling process (e.g., Fig. 2e). Though the TSV structure was only hit partially in this particular experiment, a larger $\mathrm{Cu}$ signal relative to the measurement shown in Fig. 2e is observed, where the feature was correctly targeted. To some extent, this may be explained by an inaccurate integration of the signal due to poor quality of the spectra, arising from strong space charge effects during the measurement. Beside this, it may also be that detector saturation 
occurred during the measurement, which is induced by the overwhelming host signal in this specific ablation campaign. Since the measurement presented in Fig. $2 \mathrm{f}$ was performed at lower laser power, a larger detection sensitivity is expected for $\mathrm{Cu}$, which is measured after $\mathrm{Si}\left(\mathrm{TOF}_{\mathrm{Cu}}>\mathrm{TOF}_{\mathrm{Si}}\right)$. Additionally, the expected decreasing trend of the $\mathrm{Cu}$ signal may be compensated in the course of the measurement through the enlargement of the accessible $\mathrm{Cu}$ ablation area that is mainly hit by the less intense side part of the laser beam due the particular offset between laser beam and the TSV. The measurements here are though intended to show a qualitative trend and are not considered for any quantitative purposes.

Obviously, there are still inaccuracies present with regard to sample positioning by the $\mathrm{x}-\mathrm{y}$ translation stage and the laserbeam positioning at the level of $\mu \mathrm{m}$ despite the improvements already made by the implementation of the optical microscope into the LIMS chamber. The sample positioning is typically performed in two steps. The desired target is first located with the microscope at its focal point. The sample is then laterally displaced by a well-defined (calibrated) distance until it is situated below the focal point of the laser system. Note that results obtained from non-satisfying depth profiles due to such inappropriate sample/laser positioning were not considered for further data analysis and construction of chemical depth profiles (except for the example shown in Fig. $2 \mathrm{f}$ as a proof-ofconcept). The correlation of LIMS data with actual ablation cross-sections enabled by FIB milling now allows to look for other features in the LIMS signal to be indicative for an appropriate positioning upon ablating TSVs. At this point it is important to remember that the FIB milling on the numerous investigated TSVs could only be efficiently achieved due to the etching pre-treatment which gives rise to the desired pillar-like shape.

Detection of ionized Si clusters and multiply charged Si ions. An alternative experimental approach which additionally helps identifying the critical point of crossing the $\mathrm{Si} / \mathrm{Cu} \|$ bare $\mathrm{Si}$ interface during the TSV depth profiling is based on the detection of particular ionized Si clusters and multiply charged Si ions whose yields drastically change at distinct stages of the TSVs depth profiling process. Pronounced intensity changes of these $\mathrm{Si}_{\mathrm{x}}^{+}$and $\mathrm{Si}^{\mathrm{y}}$ ions are related to the characteristics of the plasma generated inside the crater upon laser irradiation. Both plasma temperature and composition change in the course of the crater evolution thus leading to drastic changes in the signal intensities of the mass spectrometric detection of (multiple) charged $\mathrm{Si}_{\mathrm{x}}^{+}$and $\mathrm{Si}^{\mathrm{y}}$ species. In our previous LIMS compositional studies on Cu-TSVs we have observed that the generation of multiply charged $\mathrm{Si}$ ions (e.g., $\mathrm{Si}^{2+}, \mathrm{Si}^{3+}, \mathrm{Si}^{4+}$ ) is notably promoted by the presence of $\mathrm{Cu}$ in terms of a synergistic ablation effect. ${ }^{36}$ Such a facilitated $\mathrm{Si} / \mathrm{Cu}$ coablation with a characteristic 'fingerprint' in the corresponding Si mass spectra (see Fig. 4b) takes place as long as the center and high energetic part of the laser beam impacts the $\mathrm{Si} / \mathrm{Cu}$ composite (e.g., Cu-TSV embedded in surrounding Si matrix, see Fig. 4a (ii-iii)). However, characteristic changes in the Si mass spectra (Si 'fingerprint') can be noticed when the TSV bottom is reached in the course of the depth profiling process and the pre-dominant $\mathrm{Si} / \mathrm{Cu}$ co-ablation turns into a dominant Si erosion (Fig 4a (iv)). This transition leads to reduced yields of multiply charged $\mathrm{Si}$ ions (this also applies to $\mathrm{Si}^{+}$ions, see Fig. 2e) in the respective TOF mass spectra which goes along with an intensity increase for singly-charged $\mathrm{Si}$ clusters revealing larger mass-to-charge ratios (e.g., $\mathrm{Si}_{2}^{+}, \mathrm{Si}_{3}^{+} \ldots \mathrm{Si}_{7}^{+}$in Fig. 4c) ${ }^{36}$ This switch in the mass spectrometric 'fingerprint' of Si points to a transition from 'hot' to (relatively) 'cold' plasma conditions. The decrease of temperature enhances the probability of recombination of ablated species and decreases the number of charged particles. At sufficiently low temperature the collision between heavy particles (atoms and ions) becomes more important and the formation of molecular species occurs. ${ }^{37}$ A prime example thereof is demonstrated in Fig. 5a showing characteristic intensity changes of $\mathrm{Si}^{3+}$ and $\mathrm{Si}_{3}^{+}$ ions and their corresponding $\mathrm{Si}_{3}^{+} / \mathrm{Si}^{3+}$ ratio upon reaching the

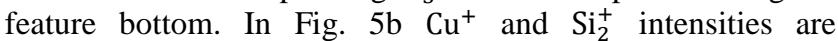
normalized with respect to $\mathrm{Si}_{3}^{+}$and $\mathrm{Si}^{3+}$ intensities and represented by red and green data points, respectively. a

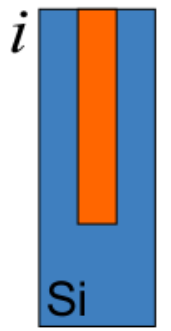
Cu-TSV pillar

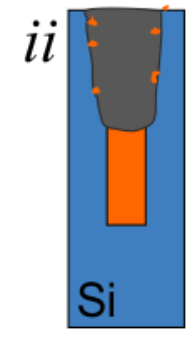

Hot plasma

b
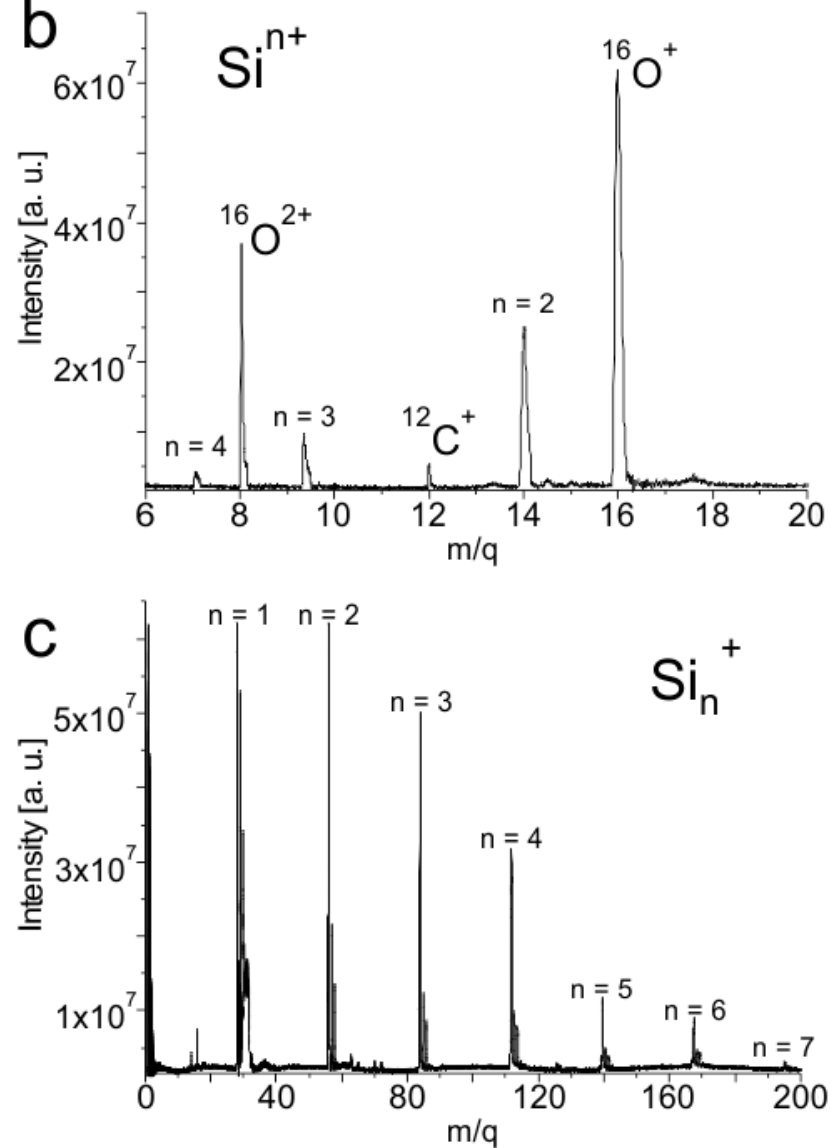

Figure 4: (a) Sketch of the ablation process of $\mathrm{Cu}-\mathrm{TSV}$ s showing distinct plasma conditions at different erosion stages $i-i v$. (b) and (c) Show single mass spectra recorded (b) 130 shots before and (c) 1'250 shots after having reached the Cu-TSV bottom of the 
structure shown in Fig 3a and 3c, which are expected to be formed at the erosion stage $i i / i i i$ and $i v$, respectively. Whereas the mass spectrum in (b) shows many multiply charged species, the mass spectrum in (c) is characterized by the formation of intensive clusters, which clearly demonstrates the different chemical nature of the occurring plasma.

Note that the $\mathrm{Si}_{2}^{+}$ion was used as $\mathrm{Si}$ tracer (instead of $\mathrm{Si}^{+}$) to avoid occasional signal saturation of the latter in its mass spectrometric detection. Although the $\mathrm{Cu}^{+}$signal intensity decreases by about 4 orders of magnitude in this representation, this drop down in intensity spans over a large number of laser pulses $(\sim 450)$ before reaching the noise level. The observed broadness in the intensity changes of the anti-correlated $\mathrm{Cu}$ and Si signals is actually unexpected considering the rather sharp and spatially confined $\mathrm{Si} / \mathrm{Cu} \|$ bare Si interface (see Figs. 3d and 4a (i)). ${ }^{38-40}$ This observation can therefore not be rationalized by particular intermixing phenomena at distinct depths reported for others binary systems ${ }^{22}$ since the entire $\mathrm{Cu}$ overburden and a certain fraction of the TSVs were removed by the mechanical polishing treatment prior to the depth profiling. Moreover, the laser beam diameter is larger than the one of the Cu-TSV. From these considerations one can safely conclude that there is an extra source of $\mathrm{Cu}$ ions contributing to the depth profiles even after passing the $\mathrm{Si} / \mathrm{Cu} \|$ bare $\mathrm{Si}$ interface. Further experimental support for this hypothesis comes from the signal evolution of $\mathrm{SiCu}^{+}$ions (Fig. 5c), whose production yield follows a similar trend as the ones of $\mathrm{Si}_{2}^{+}, \mathrm{Si}_{3}^{+} \ldots \mathrm{Si}_{6}^{+}$species. In particular, the signal intensity of this heterogeneous di-atomic cluster undergoes a noticeable rise that sets in when the $\mathrm{Si} / \mathrm{Cu} \|$ bare $\mathrm{Si}$ interface is reached. The $\mathrm{SiCu}^{+}$ion intensity passes a maximum before again dropping down to the noise level (Fig. 5c). Note however, that the intensity increase of $\mathrm{SiCu}^{+}$ions sets in at slightly lower depth than the steady decrease of the $\mathrm{Cu}^{+}$ion signal (left edge of the yellow bar in Fig. 5c).

Previous studies have revealed that the plasma expanding outwards from the ablation crater after the initial impact of the laser pulse might not be quantitatively ejected from the crater. Instead, a certain fraction of the ablated material might be redeposited at the crater side-walls, in particular when the crater depth exceeds a critical value. ${ }^{31,41}$ This $(\mathrm{Cu})$ material is preferentially trapped in the (Si) grooves that typically form at upper crater side-walls upon crater evolution (Fig. 3c and 3d). Previous LA-ICP-MS studies have already demonstrated that both the crater side-walls and the zone next to the actual ablation crater undergo certain morphological modifications due to repetitive melting and particle re-deposition during the crater evolution upon continuous laser irradiation..$^{33,42,43}$ In these studies, the particular aspect ratio of the evolving ablation crater has been identified as one key parameter among others determining the degree of morphological changes mediated by those re-deposition phenomena. From these considerations it also becomes evident that the material re-deposited at the crater side-walls is prone to continued laser ablation thus resulting in continuous re-ablation of re-deposited material in terms of a secondary ablation process superimposed on the primary uptake of target material from the crater bottom. For the ultimate quantification (e.g., of organic impurities inside the $\mathrm{Cu}-\mathrm{TSV}$ ) of laser ablation depth profiling data, it is of imminent importance that the elements detected and quantified in the mass spectrometry during the laser ablation depth profiling originate not only from the bottom of the ablation crater but to a minor extent also from the crater side-walls. This scenario is fully consistent with the smooth and slow decrease of the $\mathrm{Cu}^{+}$intensity and the anti-correlated rise in intensity of Cu-containing clusters (e.g., $\mathrm{SiCu}^{+}, \mathrm{Si}_{2} \mathrm{Cu}^{+}, \mathrm{Si}_{3} \mathrm{Cu}^{+}, \mathrm{Cu}_{2}^{+}$) that takes place when the bottom of the $\mathrm{Cu}$ via is reached (Fig. $5 \mathrm{c}$ ). Note that material re-deposition and subsequent re-ablation from the crater sidewalls have already been investigated in previous studies on the basis of optical microscopy investigations. ${ }^{31,33,43}$ However, to the best of our knowledge, no direct chemical evidence has been provided so far determining the chemical nature of the material re-deposited at the crater side walls.
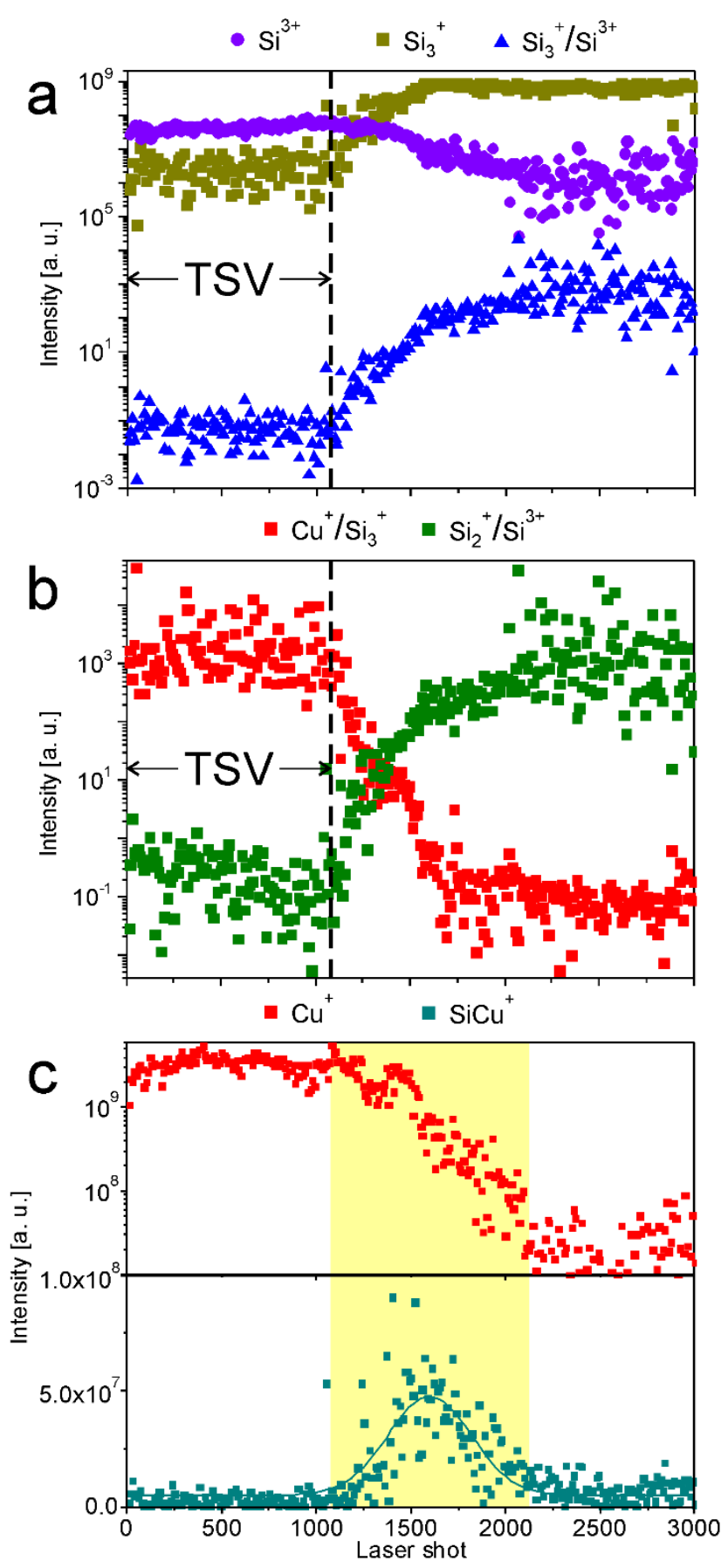

Figure 5: Chemical depth profiles of selected ions and ion intensity ratios. Experimental conditions: 3'000 laser pulses at $2.0 \mu \mathrm{J}$ pulse energy and $1 \mathrm{kHz}$ repetition rate. The dotted lines in (a) and (b) and the left edge of the yellow area in (c) indicate the location of the $\mathrm{Si} / \mathrm{Cu}-\mathrm{TSV} \|$ bare Si interface. 
EDX analysis of re-deposited material. To fill this abovementioned experimental gap, systematic inspection of the crater side-walls was carried out by means of EDX analysis. Only those samples were considered for this investigation which actually showed a successful and complete depth profiling of the TSVs in their respective cross-sectional SEM crater analysis (see e.g., Fig. 3a and 3c). A representative example of such an analysis is shown in Fig. 6 . The crater was created by applying 1 '000 laser shots at $2.44 \mu \mathrm{J}$. Besides the characteristic ripples at the crater opening and the columnar features protruding from the crater side-walls (Fig. 6a), randomly dispersed sub- $\mu \mathrm{m}$ particles can be observed in the grooves between the columnar features (Fig. 6b). The two upper panels in Fig. 6c show representative EDX point spectra recorded on the features labelled 1 and 2 in Fig. $6 b$ (recorded without SEM scanning as the beam was parked to the indicated spots).
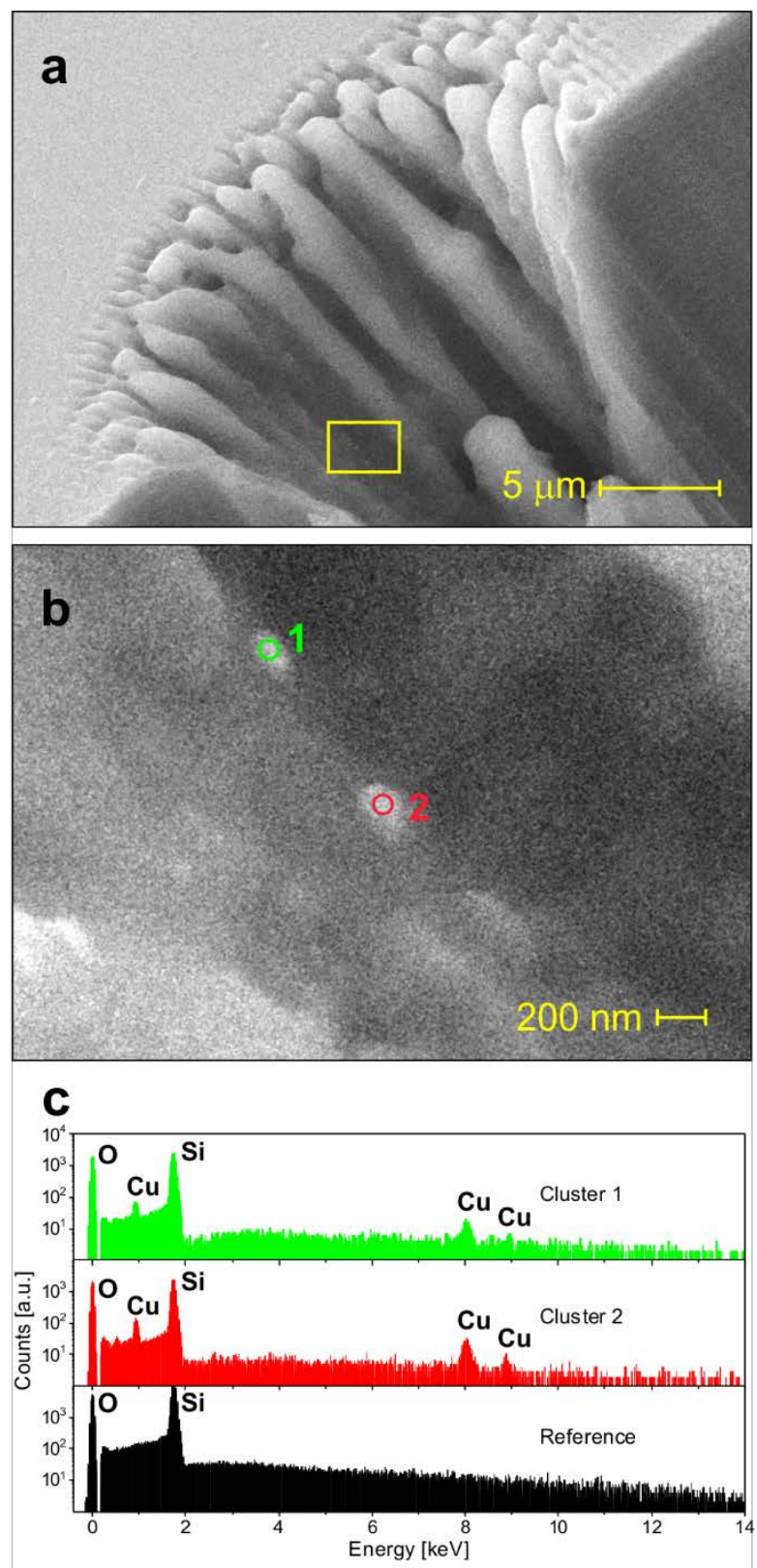

Figure 6: (a) SEM image of a laser-induced crater generated by 1'000 laser pulses at $2.44 \mu \mathrm{J}$ on a Si/Cu-TSV pillar. (b) Close-up of the crater sidewalls at the same position indicated by the yellow rectangle. (c) EDX measurements conducted on the targeted $\mathrm{Cu}$ features 1 and 2 and on a reference point on the Si host.
The most intense signals correspond to Si lines originating from the surrounding and underlying Si matrix. However, in addition to the $\mathrm{Si}$ peak, weaker but yet discernible $\mathrm{Cu}$ signals were detected. For comparison, an EDX point spectrum of the $\mathrm{Cu}$ free region on a Si crater side-wall is shown in the lower panel of Fig. 6c (black spectrum), which contains no $\mathrm{Cu}$ signal. We therefore assign the features observed in the high-resolution SEM analysis to Cu material re-deposited at the (Si) crater sidewalls. Their presence explains why the Cu-related intensities in the respective depth profiles (e.g., see Fig. 5) do not abruptly decrease when passing the delta-like $\mathrm{Si} / \mathrm{Cu} \|$ bare $\mathrm{Si}$ interface. The observed broad transition in the LIMS depth profiles can therefore be rationalized (at least partially) by these extra sidewall effects which also contribute to the mass spectrometric detection of target material during the depth profiling. These side-wall effects are also the physical origin of the temporal appearance of $\mathrm{SiCu}^{+}$species (Fig. 5c). We assume that the amount of re-deposited $\mathrm{Cu}$ on the crater side-walls reaches its maximum when the bottom of the Cu-TSV feature is reached. The amount of (re-deposited) Cu material that gets ablated from the crater side-walls right after passing the feature bottom is still sufficient to maintain 'hot' plasma conditions in terms of the above mentioned synergistic ablation effect when $\mathrm{Si}$ and $\mathrm{Cu}$ are co-ablated (Fig. 4a). Accordingly, the formation of $\mathrm{Cu}^{+}$is still favored and maintains a stable intensity level right after reaching the $\mathrm{Si} / \mathrm{Cu} \|$ bare $\mathrm{Si}$ interface while at the same time the intensity of the $\mathrm{SiCu}^{+}$related signal monotonously raises (Fig. 5c). This intermediate transition phase spans over a regime of $\sim$ 400 laser shots. The passing of the intensity maximum for $\mathrm{SiCu}^{+}$coincides with the onset of the more pronounced intensity decrease for the $\mathrm{Cu}^{+}$species.

The following monotonous intensity decrease for all $\mathrm{Cu}$ containing ions (e.g., $\mathrm{Si}_{2} \mathrm{Cu}^{+}, \mathrm{Si}_{3} \mathrm{Cu}^{+}, \mathrm{Cu}_{2}^{+}$ions) is indicative for the continuous depletion of $\mathrm{Cu}$ at the crater bottom and the crater sidewalls. This process is completed when the noise level in the respective mass spectra is reached. Along with the gradual disappearance of $\mathrm{Cu}$ containing species, the mass spectra become dominated by the parent $\mathrm{Si}^{+}$and its larger clusters (see Fig. 4c) thus being indicative for the above mentioned transition from 'hot' to 'cold' plasma conditions.

\section{Conclusions}

High-aspect ratio TSV interconnects are of general relevance for the development of future three-dimensional very large scale integration (3D-VLSI) technologies. Herein we introduced an improved preparation protocol for model TSV samples which allowed for their spatially resolved chemical analysis by means of advanced laser ablation/ionization mass spectrometry (LIMS). This novel preparation procedure yielded isolated core-shell pillars with Cu-TSV cores $\left(\varnothing_{\mathrm{TSV}}=5 \mu \mathrm{m}\right)$ embedded into spatially confined Si matrices $\left(\varnothing_{\mathrm{Si}}=50 \mu \mathrm{m}\right)$ of $\sim 80 \mu \mathrm{m}$ depth. These isolated pillar specimens served as starting point for the actual LIMS depth profiling analysis of the $\mathrm{Cu}-\mathrm{TSV}$ structures. It was this particular sample geometry which tremendously facilitated the slicing of the depth-profiled pillars by FIB milling and the subsequent cross-sectional SEM/EDX crater analysis. In the near future we will optimize the microscope camera and the translation stage systems. This will increase the targeting precision of our LIMS system and any further extensive crater treatment will not be necessary (e.g. etching and ion milling for crater cross-sections).

Our study clearly proved continuous re-deposition of ablated $\mathrm{Cu}$ material on the Si crater side-walls $\left(\varnothing_{\text {beam }}>\varnothing_{\mathrm{Cu}-\mathrm{TSV}}\right)$ during 
the laser ablation process. Even after passing the $\mathrm{Si} / \mathrm{Cu} \|$ bare $\mathrm{Si}$ interface at the feature bottom, $\mathrm{Cu}$ can be detected to some extent in the further depth profiling experiment due to the reablation of such re-deposited $\mathrm{Cu}$ material. These non-desired secondary ablation processes (side-wall effects) superimposed on the primary material uptake at the crater bottom need to be unavoidably taken into consideration for future in depth analyses of the contamination level inside the TVSs. In addition, we reported in this study a novel and robust mass spectrometric 'fingerprint' indicating the passing of the $\mathrm{Si} / \mathrm{Cu} \|$ bare Si interface during the TSV depth profiling. This 'fingerprint' is based on a pronounced change in the mass spectrometric characteristics of Si that change from spectra dominated by multiply charged (monomeric) Si ions (e.g., $\mathrm{Si}^{2+}, \mathrm{Si}^{3+}, \mathrm{Si}^{4+}$ : 'hot' plasma conditions) which are representative for the $\mathrm{Si} / \mathrm{Cu}$ co-ablation to spectra dominated by singly charged $\mathrm{Si}$ clusters (e.g., $\mathrm{Si}_{2}^{+}, \mathrm{Si}_{3}^{+} \ldots \mathrm{Si}_{6}^{+}$: 'cold' plasma conditions).

\section{AUTHOR INFORMATION}

\section{Corresponding Authors}

* E-Mail: pavel.moreno@dcb.unibe.ch, peter.broekmann@dcb.unibe.ch Phone: +41316314269.

\section{Author Contributions}

The manuscript was written through contributions of all authors. / All authors have given approval to the final version of the manuscript.

\section{ACKNOWLEDGMENT}

We acknowledge the work from the technical staff of the Department of Chemistry and Biochemistry and the Space Research and Planetary Sciences division at the University of Bern, Switzerland as well as technical staff of the Binnig and Rohrer Nanotechnology Center (U. Drechsler, S. Reidt) at IBM Research - Zurich. This work was founded by the Swiss National Science Foundation (SNSF).

\section{REFERENCES}

(1) Motoyoshi, M. Proceedings of the IEEE 2009, 97, 43.

(2) Moffat, T. P.; Wheeler, D.; Huber, W. H.; Josell, D. Electrochemical and Solid-State Letters 2001, 4, C26.

(3) Radisic, A.; Lühn, O.; Philipsen, H. G. G.; El-Mekki, Z.; Honore, M.; Rodet, S.; Armini, S.; Drijbooms, C.; Bender, H.; Ruythooren, W. Microelectronic Engineering 2011, 88, 701.

(4) Kondo, K.; Yonezawa, T.; Mikami, D.; Okubo, T.; Taguchi, Y.; Takahashi, K.; Barkey, D. P. Journal of The Electrochemical Society 2005, 152, H173.

(5) Lühn, O.; Van Hoof, C.; Ruythooren, W.; Celis, J. P. Electrochim. Acta 2009, 54, 2504.
(6) Tsai, T. C.; Tsao, W. C.; Lin, W.; Hsu, C. L.; Lin, C. L.; Hsu, C. M.; Lin, J. F.; Huang, C. C.; Wu, J. Y. Microelectronic Engineering 2012, 92, 29.

(7) De Wolf, I.; Croes, K.; Varela Pedreira, O.; Labie, R.; Redolfi, A.; Van De Peer, M.; Vanstreels, K.; Okoro, C.; Vandevelde, B.; Beyne, E. Microelectronics Reliability 2011, 51, 1856.

(8) An, J.; Moon, K. J.; Lee, S.; Lee, D. S.; Yun, K.; Park, B. L.; Lee, H. J.; Sue, J.; Park, Y. L.; Choi, G.; Kang, H. K.; Chung, C. In 2012 IEEE International Interconnect Technology Conference 2012, p 1.

(9) Grimaudo, V.; Moreno-García, P.; Cedeño López, A.; Riedo, A.; Wiesendanger, R.; Tulej, M.; Gruber, C.; Lörtscher, E.; Wurz, P.; Broekmann, P. Analytical Chemistry 2018.

(10) Grimaudo, V.; Moreno-García, P.; Riedo, A.; Neuland, M. B.; Tulej, M.; Broekmann, P.; Wurz, P. Anal. Chem. 2015, 87, 2037.

(11) Riedo, A.; Grimaudo, V.; MorenoGarcia, P.; Neuland, M. B.; Tulej, M.; Wurz, P.; Broekmann, P. J. Anal. At. Spectrom. 2015, 30, 2371.

(12) Moreno-García, P.; Grimaudo, V.; Riedo, A.; Tulej, M.; Wurz, P.; Broekmann, P. Rapid Communications in Mass Spectrometry 2016, 30, 1031.

(13) Moreno-García, P.; Grimaudo, V.; Riedo, A.; Tulej, M.; Neuland, M. B.; Wurz, P.; Broekmann, P. Electrochimica Acta 2016, 199, 394.

(14) Riedo, A.; Grimaudo, V.; MorenoGarcía, P.; Neuland, M. B.; Tulej, M.; Broekmann, P.; Wurz, P. CHIMIA International Journal for Chemistry 2016, 70, 268.

(15) Grimaudo, V.; Moreno-García, P.; Riedo, A.; Meyer, S.; Tulej, M.; Neuland, M. B.; Mohos, M.; Gütz, C.; Waldvogel, S. R.; Wurz, P.; Broekmann, P. Analytical Chemistry 2017, 89, 1632.

(16) Moenke-Blankenburg, L. Prog. Anal. Spectroscop. 1986, 9, 335.

(17) Koch, J.; Günther, D. Applied Spectroscopy 2011, 65, 155A.

(18) Hahn, D. W.; Omenetto, N. Applied Spectroscopy 2010, 64, 335A.

(19) Grimaudo, V.; Moreno-García, P.; Cedeño López, A.; Riedo, A.; Wiesendanger, R.; 
Tulej, M.; Gruber, C.; Lörtscher, E.; Wurz, P.; Broekmann, P. Anal. Chem. 2017, in press.

(20) Moffat, T. P.; Wheeler, D.; Edelstein, M. D.; Josell, D. IBM J. Res. Dev. 2005, 49, 19.

(21) West, A. C.; Mayer, S.; Reid, J. Electrochem. Solid-State Lett. 2001, 4, C50.

(22) Cedeño López, A.; Grimaudo, V.; Moreno-García, P.; Riedo, A.; Tulej, M.; Wiesendanger, R.; Wurz, P.; Broekmann, P. J. Anal. At. Spectrom. 2018, in press.

(23) Rohner, U.; Whitby, J. A.; Wurz, P. Meas. Sci. Technol. 2003, 14, 2159.

(24) Riedo, A.; Bieler, A.; Neuland, M.; Tulej, M.; Wurz, P. J. Mass Spectrom. 2013, 48, 1.

(25) Riedo, A.; Neuland, M.; Meyer, S.; Tulej, M.; Wurz, P. J. Anal. At. Spectrom. 2013, 28, 1256.

(26) Riedo, A.; Meyer, S.; Heredia, B.; Neuland, M. B.; Bieler, A.; Tulej, M.; Leya, I.; Iakovleva, M.; Mezger, K.; Wurz, P. Planet. Space Sci. 2013, 87, 1.

(27) Neuland, M. B.; Meyer, S.; Mezger, K.; Riedo, A.; Tulej, M.; Wurz, P. Planet. Space Sci. 2014, 101, 196.

(28) Tulej, M.; Iakovleva, M.; Leya, I.; Wurz, P. Analytical and Bioanalytical Chemistry 2010, 399, 2185.

(29) Wiesendanger, R.; Wacey, D.; Tulej, M.; Neubeck, A.; Ivarsson, M.; Grimaudo, V.; Moreno-Garcia, P.; Cedeño López, A.; Riedo, A.; Wurz, P. Astrobiology 2018, Submitted.

(30) Meyer, S.; Riedo, A.; Neuland, M. B.; Tulej, M.; Wurz, P. Journal of Mass Spectrometry 2017, 52, 580.

(31) Bonse, J.; Baudach, S.; Krüger, J.; Kautek, W.; Lenzner, M. Applied Physics A 2002, 74, 19.
(32) Finn, D. S.; Lin, Z.; Kleinert, J.; Darwin, M. J.; Zhang, H. Journal of Laser Applications 2015, 27, 032004.

(33) Eggins, S. M.; Kinsley, L. P. J.; Shelley, J. M. G. Applied Surface Science 1998, 127, 278.

(34) Guosheng, Z.; Fauchet, P. M.; Siegman, A. E. Physical Review B 1982, 26, 5366.

(35) Seifert, G.; Kaempfe, M.; Syrowatka, F.; Harnagea, C.; Hesse, D.; Graener, H. Applied Physics A 2005, 81, 799.

(36) Grimaudo, V.; Moreno-García, P.; Cedeño López, A.; Riedo, A.; Wiesendanger, R.; Tulej, M.; Gruber, C.; Lörtscher, E.; Wurz, P.; Broekmann, P. Anal. Chem. 2017, Submitted.

(37) Alessandro De, G.; Jörg, H. Journal of Physics D: Applied Physics 2017, 50, 183002.

(38) He, M.; Li, B.; Yu, S.; Zhang, B.; Liu, Z.; Hang, W.; Huang, B. Journal of Analytical Atomic Spectrometry 2013, 28, 499.

(39) Garcia, C. C.; Vadillo, J. M.; Palanco, S.; Ruiz, J.; Laserna, J. J. Spectrochimica Acta Part B: Atomic Spectroscopy 2001, 56, 923.

(40) Ardakani, H. A.; Tavassoli, S. H. Spectrochimica Acta Part B: Atomic Spectroscopy 2010, 65, 210.

(41) Lowndes, D. H.; Fowlkes, J. D.; Pedraza, A. J. Applied Surface Science 2000, 154, 647.

(42) Borisov, O. V.; Mao, X.; Russo, R. E. Spectrochimica Acta Part B: Atomic Spectroscopy 2000, 55, 1693.

(43) J. G. Mank, A.; R. D. Mason, P. Journal of Analytical Atomic Spectrometry 1999, 14, 1143. 


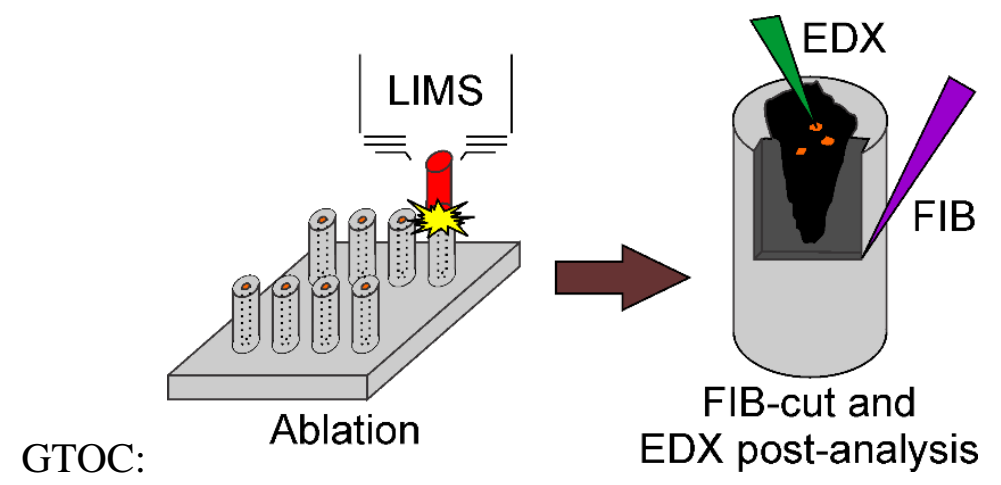

